\title{
Ecchymoma to Liver Cirrhosis: Report of a Case and a Review of the Literature
}

\author{
Long Gao, Wei-yu Wu, Yan Zhou, Ming-li Wu and Xiao-an Li*
}

Department of Gastroenterology, The Gastroenterology Tumor and Microenvironment Laboratory, The First Affiliated Hospital of Chengdu Medical College, Chengdu Medical College, Chengdu, P.R. China

\begin{abstract}
Ecchymoma in a patient with unclear liver cirrhosis was rare. Herein we reported such a case of a 67-year-old female with cirrhosis and with a huge ecchymoma developed due to a slightly cough. She felt tired with strong left abdominal pain after a slightly cough that happened 3 days before. After a detailed diagnosis, she was believed to be a case of ecchymoma caused by liver cirrhosis. And a conservative therapy strategies that introducing blood plasma into body and enhancing hemostasia to protect hepatocytes had been considered. Although ecchymoma with cirrhosis was rare, an appropriate treatment plan should be discussed on a case-by-case basis to improve the prognosis.
\end{abstract}

Keywords: Ecchymoma; Cirrhosis; Therapy strategies

\section{Introduction}

Liver cirrhosis was commonly associated with coagulopathies, including thrombocytopenia and hypoprothrombinemia, which often cause easy bruising and bleeding [1]. Sudden bleeding from gastrointestinal varices due to portal hypertension was also an established risk of liver cirrhosis.

However, hematoma was an uncommon condition of liver cirrhosis [2]. Only a few cases had been reported. Tomoo [3] firstly described two cases of subdural hematoma as a complication of liver cirrhosis due to thrombocytopenia and coagulation deficiency. However, both patients were died of complications in hospitalization. Masaaki Takamura [4] reported three cases of alcoholic cirrhosis complicated by deep bleeding into the muscles or retroperitoneum. All of patients were died one month after the onset of this rarely ecchymoma complication that causing liver failure. Suguru Yamashita [5] reported a case of a patient with alcoholic liver cirrhosis who underwent iliopsoas muscle hematoma and subsequently died in the hospital after emergency surgery therapy. Hematoma had a high mortality so we should cautiously make a diagnosis and treatment.

We herein presented another a case that patient with Child-Pugh score $\mathrm{C}$ cirrhosis and treated conservatively. Fortunately, the patient recovered within one month after conservative treatment. Although rare, it was a complication with a poor prognosis, and a treatment plan should be carefully determined.

\section{Case Report}

The patient was a 67-year-old female. She had no a history of virus-related hepatitis, immune abnormality, chronic alcoholism, schistosomiasis, industrial poison and medicine, cholestasis, inherited metabolic diseases, Budd-Chiari syndrome. She suffered from abdominal pain after a light cough that had happened 3 days before. She then visited a local physician as she developed a sense of weakness of body and persisted pain of abdomen, after a primary examination, the doctor suspected she was suffered from liver cirrhosis and transferred the patient to our hospital. Gradually, she developed worsening anemia, dyspnea and weakness. From the result of physical examination, we found she looked dizzy, exhausted easily, pale face, dullness to percussion in her right lung. What's more, although not any ecchymosis been observed, on the left side of her abdomen could be seen an abdominal mass which had an unclear boundary, obviously tenderness, rebound tenderness and mascular tension. Besides, both lower extremities showed mild pitting edema.
On admission, she was weakness and with a blood pressure of $119 / 74 \mathrm{mmHg}$ and a heart rate of $104 \mathrm{bpm}$. Her laboratory findings included Hct 27.6\%, Hb 95 g/L, RBC 3.02×109/L, PLT 110×109/L. Blood urea was $3.98 \mathrm{mmol} / \mathrm{L}$ (normal<8.2 mmol/L), creatinine $64.4 \mu \mathrm{mol} / \mathrm{L}$ (normal $<84 \mu \mathrm{mol} / \mathrm{L}$ ), aspartate aminotransferase $326 \mathrm{U} / \mathrm{L}$ (normal $<35$ $\mathrm{U} / \mathrm{L}$ ), alanine aminotransferase $234 \mathrm{U} / \mathrm{L}$ (normal $<40 \mathrm{U} / \mathrm{L}$ ), alkaline phosphatase $176 \mathrm{U} / \mathrm{L}$ (normal<130 U/L) gamma-glutamyltransferase $196 \mathrm{U} / \mathrm{L}$ (normal $<45 \mathrm{U} / \mathrm{L}$ ), total bilirubin $56.2 \mu \mathrm{mol} / \mathrm{L}$ (normal $<28$ $\mu \mathrm{mol} / \mathrm{L}$ ), prothrombin time $15.0 \mathrm{sec}$ (INR 1.39), fibrinogen $1.36 \mathrm{~g} / \mathrm{L}$, total protein $56.1 \mathrm{~g} / \mathrm{L}$ (normal $>65 \mathrm{~g} / \mathrm{L}$ ), albumin $25.8 \mathrm{~g} / \mathrm{dl}$ (normal $>40$ $\mathrm{g} / \mathrm{L}$ ), globulins $30.3 \mathrm{~g} / \mathrm{L}$ (normal $>20 \mathrm{~g} / \mathrm{L}$ ), AFP $102 \mathrm{ng} / \mathrm{mL}$ (normal<20 $\mathrm{ng} / \mathrm{mL}$ ), CEA $5.27 \mathrm{ng} / \mathrm{mL}$ (normal<5 ng/mL), ferritin: $226.5 \mathrm{ng} / \mathrm{mL}$ (normal<219 ng/mL) CA-125: 75.1 U/Ml (normal<35.0 U/mL), CA$199142.6 \mathrm{U} / \mathrm{Ml}$ (normal<35.0 U/mL), HBV Ab $39.61 \mathrm{mIU} / \mathrm{mL}, \mathrm{LDH}$ $303 \mathrm{U} / \mathrm{L}$ (normal<214 U/L), a-HBDH $234 \mathrm{U} / \mathrm{L}$ (normal<182 U/L), Lipase $65 \mathrm{U} / \mathrm{L}$ (normal<60 U/L). Electrolytic, cardiac protein spectrum, feces routine, routine urine test, CRP, ESR, tuberculosis antibody, antinuclear antibodies, rheumatoid factor, anticardiolipin antibodies, anti-smooth muscle antibodies, antimitochondrial and antiparietal cell antibodies were all negative.

In contrast-enhanced CT performed: left lower abdomen was enlarged with a huge hematoma with a maximal diameter of $4.5 \mathrm{~cm}$ accompanied by formation of fluid level, and the border of the hematoma was not clear (Figure 1); severe hepatocirrhosis, hypersplenotrophy, peritonitis, seroperitoneum were also observed, and at the right lobe of liver, a large areas of low density was observed which in arterial phase and delayed phase exhibited heterogeneous radiography; uncinate process of the pancreas seemed swelling and vague with the descending duodenum (Figure 2). Color Doppler Ultrasound performed bilateral pleural effusion (Larger in right side); left lower abdomen: Echo anomaly, hematoma possibly (Figure 3).

*Corresponding author: Xiao-an Li, Department of Gastroenterology, The Gastroenterology Tumor and Microenvironment Laboratory, The First Affiliated Hospital of Chengdu Medical College, Chengdu Medical College, Chengdu, P.R. China, Tel: +86-28-83016968; E-mail: lixa@sari.ac.cn

Received November 07, 2015; Accepted December 18, 2015; Published December 26 , 2015

Citation: Gao L, Wu W, Zhou Y, Wu M, Li X (2015) Ecchymoma to Liver Cirrhosis: Report of a Case and a Review of the Literature. J Clin Case Rep 5: 671. doi:10.4172/2165-7920.1000671

Copyright: @ 2015 Gao L, et al. This is an open-access article distributed under the terms of the Creative Commons Attribution License, which permits unrestricted use, distribution, and reproduction in any medium, provided the original author and source are credited. 

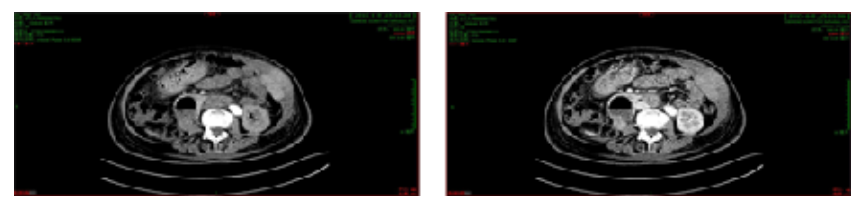

Figure 1: Enhanced abdominal CT showed that the abdomen were enlarged with huge hematoma accompanied by formation of fluid level (A: arterial phase, B: venous phase ).
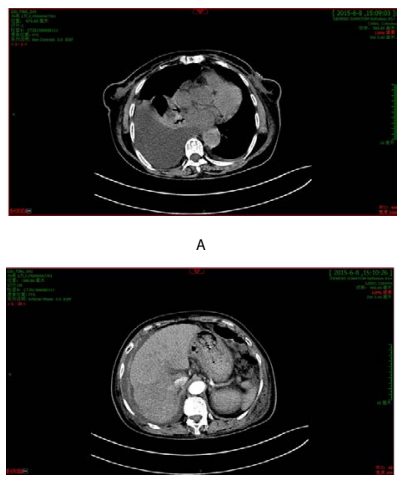

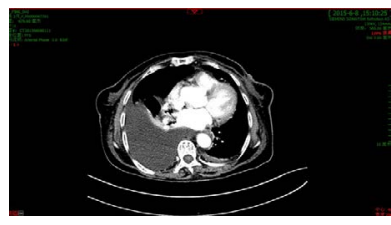

B

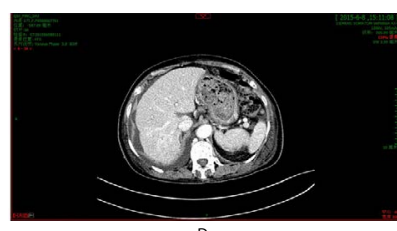

Figure 2: Severe hepatocirrhosis(C: arterial phase, D: venous phase) and hypersplenotrophy, peritonitis, seroperitoneum(A: delayed phase, B: arterial phase) were observed in contrast-enhanced CT.

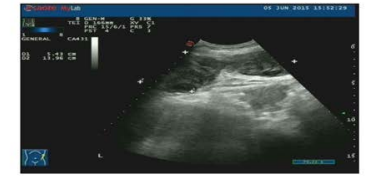

A

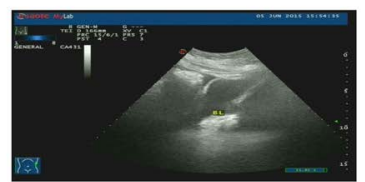

C

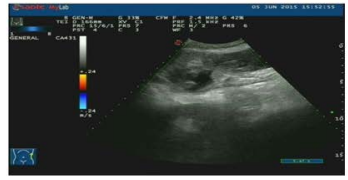

B

D

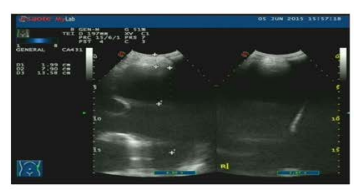

Figure 3: Color Doppler Ultrasound performed bilateral pleural effusion in left lower abdomen, which hematoma possibly.

Continuously low flow oxygen and emergency administering red cell concentrates were admitted to the patients immediately after transferred to our hospital. Before pleural effusion, two kinds of antibiotics treatment were started. Glutathione and Diisopropylamine Dichloroacetate were both used to protect the liver. To ameliorate hemostatic deficiency, fresh frozen plasma and vitamine K were injected to the patient every day. Somatostatin, abdominal bandage compression and hemocoagulase were used to enhance the effect of the hemostasis. Saline was restricted in $1 \mathrm{~L}$, human serum albumin and furosemide (20 $\mathrm{mg}$ /day) combined spirolactone $(60 \mathrm{mg} /$ day $)$ were treated to increase the secretion of urine which can relieve parapneumonic effusion and pyoperitoneum. After making paracentesis of chest (at day of 4 and 8), we diagnosis the patient suffered from filling the chest with transudate hydrothorax.

At first, we suspected she suffered from liver cirrhosis which the severity of LC was classified as Child-Pugh grade C. What was worse, on the 4 day of admission, her overall condition continued to deteriorate and felt breathing difficult. Hemoglobin decreased to $75 \mathrm{~g} / \mathrm{L}$, Hct $22.3 \%$, RBC $2.39 \times 1012 \mathrm{~g} / \mathrm{L}$, and there was a large area of ecchymosis could be observed in left abdomen, perineal and waist. Reexamining the function of Coagulation and liver indicated: prothrombin time $13.4 \mathrm{sec}$ (INR 1.24), fibrinogen $1.88 \mathrm{~g} / \mathrm{L}$, D-dimer $889.0 \mathrm{ng} / \mathrm{mL}$ (normal <255 ng/mL), FDP:5.74 ug/mL; creatinine 64.4 $\mu \mathrm{mol} / \mathrm{L}$, aspartate aminotransferase $102 \mathrm{U} / \mathrm{L}$, alanine aminotransferase $120 \mathrm{U} / \mathrm{L}$, alkaline phosphatase $124 \mathrm{U} / \mathrm{L}$ gamma-glutamyltransferase $155 \mathrm{U} / \mathrm{L}$, total bilirubin $65.7 \mu \mathrm{mol} / \mathrm{L}$, total protein $56.5 \mathrm{~g} / \mathrm{L}$, albumin $26.5 \mathrm{~g} / \mathrm{dl}$ (normal $>40 \mathrm{~g} / \mathrm{L}$ ), globulins $30.3 \mathrm{~g} / \mathrm{L}$, Enlarging fresh frozen plasma infusion and using abdominal bandage compression to enhance arresting bleeding. Due to obviously uncomfortable breathing, chest puncture was used to relieve the symptoms. On the 6 day of admission, patient began to be improved and her hemoglobin became recovered to $81 \mathrm{~g} / \mathrm{L}$. In order to preventing infection, promoting the recovery of patient and clarifying the nature of the chest, we took the extraction of pleural effusion again at the day of 8 . At 22 day, her laboratory findings included, AFP:38.2 ng/mL, CEA:6.82 ng/mL, CA-199:49.6 ng/mL, CA-125: $119.9 \mathrm{ng} / \mathrm{mL}$, a normal coagulation, Hb 92 g/L, Hct 27.9\%, aspartate aminotransferase $59 \mathrm{U} / \mathrm{L}$, alanine aminotransferase $44 \mathrm{U} / \mathrm{L}$, alkaline phosphatase $116 \mathrm{U} / \mathrm{L}$, gamma-glutamyltransferase $138 \mathrm{U} / \mathrm{L}$, albumin $31.0 \mathrm{~g} / \mathrm{dl}$, globulins $33.4 \mathrm{~g} / \mathrm{L}$. There was hardly found any parapneumonic effusion and pyoperitoneum, and no obvious edema of lower limbs was been observed. The severity of LC was re-markered as Child-Pugh grade B. Owing to the economic reason; EUS-FNA was failed to diagnosis the problem of the pancreatic. Patient was followed up after hospital discharged.

\section{Discussion}

Liver cirrhosis could appear skin and mucosa petechia or ecchymosis. It was reported that a small amount of concurrent retrobulbar hematoma and retroperitoneal hemorrhage could be occurred after cirrhosis, however, there was rarely reported of cirrhosis complicated with a large area of ecchymoma. Huang et al. [6] summarized spontaneous intracranial hemorrhage (SICH) in 4515 hospitalized Chinese cirrhotic patients. Interestingly, among these patients, 36 suffered from SICH and 78\% were male. The main etiology of cirrhosis included alcoholic cirrhosis (50\%), virus-related cirrhosis (27.8\%), and combined virus- and alcohol-related cirrhosis (22.2\%). What's more, the incidence of SICH in alcohol-related cirrhosis was more than in virus-related cirrhosis. Several reasons of alcohol might lead to hemorrhage, such as the inhibition of platelet adhesion to fibrinogen [7], dose-related ethanol suppression of platelet aggregation induced by extravasation [8], and the promotion of atherosclerosis [9]. The high rate of alcohol-related cirrhotic hemorrhage was considered to be associated with dysfunctional coagulation in LC as well as dysfunctional hemostasis due to fragile vascular walls caused by alcohol. According to a report, a case of intestinal obstruction due to intramural hematoma of duodenum following therapeutic endoscopy for a bleeding duodenal ulcer in a patient with HCV-liver cirrhosis [10].

The reasons of viral-related cirrhosis trended to occur hematoma were as follows: portal hypertension, liver dysfunction, cirso-epigastrica and cirso-retroperitoneal vein, made patients increasing the tendency of epigastric vessels bleeding. At the same time, because of relatively serious destruction of coagulation dysfunction in liver cirrhosis, it was easy to cause bleeding and hematoma. Besides, irregular anti-viral treatment and poor curative effect of viral hepatitis led to vascular and nervous system damage was also been charged with hematoma [11].

However, in the case, we performed a detailed inquiry of historytaking and clinical examination, and found that there was no reason 
Citation: Gao L, Wu W, Zhou Y, Wu M, Li X (2015) Ecchymoma to Liver Cirrhosis: Report of a Case and a Review of the Literature. J Clin Case Rep 5: 671. doi:10.4172/2165-7920.1000671

for virus-related hepatitis, immune abnormality, chronic alcoholism, schistosomiasis, industrial poison and medicine, cholestasis, inherited metabolic diseases, Budd-Chiari syndrome and so on. At first we deliberated the patients might suffer with primary liver cancer. However, contrast-enhanced CT scan did not exhibit typical imaging manifestations of hepatocellular carcinoma (CT density value density was similar to fat density) and AFP did not rise up to the standard of diagnosing a liver cancer. At the same time, though we did not use any anti-tumor strategy, the level of AFP rapidly decreased and the function of liver obviously recovered which did not inconsistent with the natural course of liver cancer. Although contrast-enhanced CT scan and the clinical evaluation of tumor markers like CA199, CEA, CA125 did reminder that the possibility of pancreatic carcinoma should be considered, we did not observe any abdominal lymphatic metastasis or lymphadenectasis, and other parts of metastasis tissue. Combined with the result of endoscopic ultrasonography in our hospital and laboratory test of bilirubin, ALP and GGT which showed the pressure symptom of biliary and gastrointestinal was not serious. So the diagnosis of pancreatic related cirrhosis was not enough as well. We speculated that the main etiology of cirrhosis in the patient may unclear, or partly caused by non-alcoholic fatty liver. The patient's subcutaneous hematoma occurred may be related to the following factors: First, the severity of LC was markered as Child-Pugh grade $C$ which indicated there was a serious damage to the liver. Then vitamin $\mathrm{K}$-dependent coagulation factors such as II , VII, IX, X that mainly synthetised in liver became obviously decreased which lead to prolonging PT and making coagulation disorders. At the same time, the dysfunction of hepatocyte caused increasingly consumption of blood coagulation factors, fibrinolysis and hyperfibrinolysis.

For a patient in a decompensated cirrhosis condition, Peripheral blood vessels presented an increased angiopsathyrosis and vasopermeability [1]. As a result, red blood cells could easily leakage to the extravascular that prone to bleeding occurred in skin and mucous membrane. All of above factors increased the incidence of subcutaneous hematoma. In this case, an increased angiopsathyrosis could lead to subcutaneous hematoma after local trigger (the patients with history of coughing). Due to bleeding in a high speed and large capacity, it's could result in an obviously decrease of hypovolemia and then lead to a hemorrhagic shock. At the same time, under the condition. It was easy to induce hepatic encephalopathy, hepatorenal syndrome and other complications that caused the death of the patient.

\section{Conclusions}

When the diagnosis of a cirrhosis patient appeared local skin swelling or pain, subcutaneous hematoma should be taken into consideration. This case was presented on account of its rarity and a successfully conservative treatment strategies.

\section{References}

1. Muciño-Bermejo J, Carrillo-Esper R, Uribe M, Méndez-Sánchez N (2013) Coagulation abnormalities in the cirrhotic patient. Ann Hepatol 12: 713-724.

2. Di Bisceglie AM, Richart JM. (2006) Spontaneous retroperitoneal and rectus muscle hemorrhage as a potentially lethal complication of cirrhosis. Liver Int 26: $1291-1293$.

3. Furui T, Yamada A, Iwata W (1989) Subdural hematoma as a complication of hemostatic deficiency secondary to liver cirrhosis--report of two cases. Neurol Med Chir 29: 588-91.

4. Takamura M, Watanabe J, Sakamaki A, Honda Y, Kamimura K, et al. (2014) Alcoholic liver disease complicated by deep bleeding into the muscles or retroperitoneum: report of three cases and a review of the literature. Intern Med 53: $1763-1768$.

5. Yamashita S, Tanaka N, Nomura Y, Miyahara T, Furuya T (2012) Iliopsoas muscle hematoma secondary to alcoholic liver cirrhosis. Case Rep Gastroenterol 6: 704-711.

6. Huang HH, Lin $\mathrm{HH}$, Shih $\mathrm{YL}$ (2008) Spontaneous intracranial hemorrhage in cirrhotic patients. Clin Neurol Neurosurg 110: 253-258.

7. de Lange DW, Hijmering ML, Lorsheyd A, Scholman WL, Kraaijenhagen RJ, et al. (2004) Rapid intake of alcohol (binge drinking) inhibits platelet adhesion to fibrinogen under flow. Alcohol Clin Exp Res 28: 1562-1568.

8. Horak JK, Brandon TA, Ribeiro LG (1982) Effects of ethanol and hemolysis on in vivo and in vitro platelet aggregation. J Cardiovasc Pharmacol 4: 1037-1041.

9. Cooper DE, Goff DC Jr, Bell RA, Zaccaro D, Mayer-Davis EJ, et al. (2002) Is insulin sensitivity a causal intermediate in the relationship between alcohol consumption and carotid atherosclerosis?: the insulin resistance and atherosclerosis study. Diabetes Care 25: 1425-1431.

10. Sugai K, Kajiwara E, Mochizuki Y (2005) Intramural duodenal hematoma after endoscopic therapy for a bleeding duodenal ulcer in a patient with liver cirrhosis. Intern Med 44: 954-957.

11. Weissenborn K, Tryc AB, Heeren M, Worthmann H, Pflugrad H, et al. (2009) Hepatitis C virus infection and the brain. Metab Brain Dis 24: 197-210. 